CIEEE 2011 This paper is published in the IEEE Transactions on Information Technology in Biomedicine it is available from IEEEXplore at $<$ http://dx.doi.org/10.1109/TITB.2011.2118763>

\title{
Limb Movements Classification Using Wearable Wireless Transceivers
}

\author{
Anda R. Guraliuc, Paolo Barsocchi, Francesco Potortì, Paolo Nepa, Member, IEEE
}

\begin{abstract}
A feasibility study where small wireless transceivers are used to classify some typical limb movements used in physical therapy processes is presented. Wearable wireless low-cost commercial transceivers operating at $2.4 \mathrm{GHz}$ are supposed to be widely deployed in indoor settings and on people's bodies in tomorrow's pervasive computing environments. The key idea of this work is to exploit their presence by collecting the received signal strength measured between those worn by a person. The measurements are used to classify a set of kinesiotherapy activities. The collected data are classified using both Support Vector Machine and K-Nearest Neighbour methods, in order to recognise the different activities.
\end{abstract}

Index Terms - Classification of human limbs activities, KNearest Neighbour (K-NN), Received Signal Strength (RSS), Support Vector Machine (SVM), wearable wireless devices.

\section{INTRODUCTION}

$\mathrm{T}$ HE increasing improvements of wireless technology and miniaturized sensors made possible the proliferation of wireless sensor networks. These communications networks are one of the first real world examples of pervasive computing: they are composed of small, smart and cheap sensing devices that promise to eventually permeate the environment. We may face a not so distant future where small sensors, all capable of wireless communication, are ubiquitously deployed in the environment and on people's body.

Extensive attention has been focused in the literature on wireless sensor networks, especially in the framework of wireless body sensor networks [1]. In particular, wearable wireless systems were developed to detect, track and understand people's behaviour. In recent years, the detection of body posture and activity received a significant interest for their application in sports, medicine and military. Recognizing people's activities is also a key issue in Assisted Living (AL) applications, where it can be useful for example to rate how a person performs routine activities $[2,3]$.

One further field of application is kinesiotherapy, where the aim is to provide monitoring of physical therapies for patients who have suffered a stroke, multiple sclerosis, joint replacements or reconstructions, amputation, brain and spinal

cord injury, or some motor function disability resulting from Parkinson's disease [4-6]. For these cases, wireless body

\footnotetext{
Manuscript received...

Anda R. Guraliuc and Paolo Nepa are with the Department of Information Engineering, University of Pisa, Italy (via Caruso 16, I-56122, Pisa, Italy, phone: +39 050 2217511; fax: +39 050 2217522; e-mail: \{anda.guraliuc, paolo.nepa\}@iet.unipi.it).

Paolo Barsocchi and Francesco Potorti are with the ISTI Institute of CNR (via Moruzzi 1, I-56124, Pisa, Italy, e-mail: \{paolo.barsocchi, potorti\}@isti.cnr.it): their work is supported in part by the European Commission in the framework of the FP7 project UNIVERSAAL (contract N. 247950).
}

sensor networks could replace the existing wired telemetry systems [7], allowing remotely supervised kinesiotherapy. In a typical wearable Wireless Body Area Network (WBAN) scenario, a patient wears some sensors that form an on-body sensor network, while an off-body base station registers data collected by the WBAN. The therapy effectiveness can be evaluated by specialized medical operators performing an analysis on the registered data. Continuous remote monitoring allows the patient to have a normal life by reducing the inconvenience of regular visits to the therapist. The purpose of using a WBAN is to improve health care quality and efficacy, and also to reduce health assistance costs. Studies relative to WBANs usually include the use of various transducers like accelerometers and gyroscopes $[6,8]$. Alternative approaches include motion capture systems [9], which are based on video cameras that can follow the movements of a number of markers placed on the human body in order to reconstruct its activity. These systems are expensive and require a large number of constitutive elements (cameras and markers). Research activities were also carried out to monitor body movement during sleep, because people with sleep deficits may experience impaired performance, irritability, lack of concentration, and daytime drowsiness. This application is especially relevant for researchers interested in children or adults with dementia. In [10], movement detection and classification is performed by means of thermistors, video cameras and load cells placed at the bed frame feet.

In this paper wearable wireless transceivers were used to classify some typical limb kinesiotherapy exercises that can be performed during a rehabilitation/recovery process. Three low-cost Crossbow IRIS transceiver modules [11] placed on the limbs and abdomen plus an off-body fixed node form the wireless network. The objective is to recognise the performed activities using only the Received Signal Strength (RSS), a value that can be readily obtained from low-cost wireless communication devices. There are at least two possible scenarios where this approach, if successful, can be useful. In the first, futuristic scenario, wireless sensors are ubiquitous in the environment and on the patient's body. In this case no additional equipment is required to recognise simple limb movements. In the second scenario, various sensors (i.e. accelerometers, gyroscopes, pressure sensors) are placed on the patient's body to recognise the movements. In the common case of the sensors using wireless communications, exploiting the RSS measurements can give an additional source of information at no additional equipment cost.

A wide variety of techniques and algorithms are found in the literature to classify measurements for posture and movement recognition. Most of them are based on traces collected using accelerometers and gyroscopes. Techniques range from feed-forward back propagation neural networks [8 ] to discrete wavelet transforms [10], support vector machine (SVM) techniques [12,13] and hidden Markov models [14]. In this paper Support Vector Machine (SVM) and K-Nearest 
CIEEE 2011 This paper is published in the IEEE Transactions on Information Technology in Biomedicine it is available from IEEEXPlore at $<$ http://dx.doi.org/10.1109/TITB.2011.2118763>

Neighbour (K-NN) classification techniques were implemented to recognise different activities, due to their success in many classification problems [12,13]. Our purpose is not to present a finely tuned and well-engineered algorithm, but to show that standard classification methods have the potential to solve the problem with acceptable accuracy.

The paper is organized as follows. Section II presents the equipment architecture and some typical human body limb activities that can be used in a rehabilitation/recovery process. The two methods used to classify the limb movements are also briefly described. Results are reported and commented in Section III and concluding remarks are drawn in Section IV.

\section{Methodology}

\section{A. Equipment set-up}

The WBAN was formed by Crossbow IRIS transceivers (motes) operating at $2.4 \mathrm{GHz}$ (ISM band) according to the IEEE 802.15.4 protocol. The motes were placed on the body and a fixed off-body node was connected to a PC. The motes form an ad hoc on-body wireless network with a tree topology determined by the relative location on the body and by the wireless link characteristics (see Fig. 1). The gateway was the root of the entire network and was connected to the processing server; the TX mote was placed at the centre of the front waist and the RX motes (namely S1 and S2) were placed on either the right arm or the right leg. Each device was fixed to the arm/leg with an elastic band. The TX mote periodically sends a beacon packet with a fixed transmission power $(3 \mathrm{dBm})$. The RX motes sample the received beacon, estimate the RSS and send it to the gateway node. The sampling frequency should be chosen considering on one side the computing constraints and networking overhead - which are both direct responsible of power consumption in the sensors - and on the other side RSS waveform reconstruction accuracy. Given the relatively slow motion, it was possible to set the sampling rate to a no-compromise value of $8 \mathrm{~Hz}$.

Measurements were performed on two different healthy individuals: a $1.58 \mathrm{~m}$ height, $53 \mathrm{~kg}$ weight 25 -years female (hereafter user A) and a $1.77 \mathrm{~m}$ height, $75 \mathrm{~kg}$ weight 24-years male (hereafter user B). The environment consisted of an office room. From a wave propagation point of view, this is similar to a home environment/single room hospital environment. Big rooms or alleys exhibit different propagation characteristics, even if the experience of the authors suggests that it is unlikely that the different propagation environment does in fact make a significant difference for motes placed on body. From a point of view of interference with other devices transmitting in the $2.4 \mathrm{GHz}$ band, the office environment is more severe than a typical home environment.

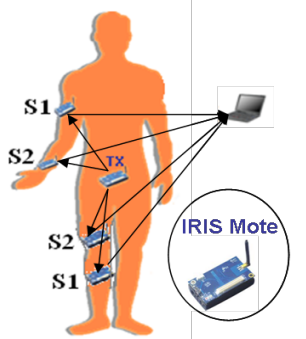

Fig. 1. Wearable wireless network. TX is the transmitter placed at the centre of the front waist, S1 and S2 are the receiving motes placed on the arm/ leg.

A serious comparison with a hospital environment would require a dedicated measurement campaign, but in the authors' judgement it is not crucial for a feasibility study.

\section{B. Arm and leg movements}

For arm activity recognition, the RX motes were placed on the right arm, one at the wrist and one on the upper part of the arm as shown in Fig. 1. For leg activity recognition, the $\mathrm{RX}$ motes were placed on the right leg, one on the thigh just above the knee and one just below the knee. The kinesiotherapy activities performed in this paper are summarized in Table I. During the measurements the subject was standing and only the arm/leg with the sensors on it was performing the activities described in Table I. Each exercise, lasting for 30 seconds, was repeated fifty times. The fifty repetitions, for both volunteers, were performed in different days, to avoid fatigue and to verify the experiment repeatability. During the measurements, the activities were performed with a slow rate of 20-30 oscillations per minute.

\section{Classification methods}

Classification is a procedure that assigns a given object to a given number of classes. A classifier is trained using a

TABLE I

SCHEMATIC REPRESENTATION OF ARM/LEG ACTIVITIES CONSIDERED DURING THE

\begin{tabular}{|c|c|c|c|}
\hline \multicolumn{4}{|c|}{ ANALYSIS } \\
\hline Activity1 & $\begin{array}{l}\text { oscillating from } \\
\text { straight down } \\
\text { (parallel to body) to } \\
\text { horizontal straight } \\
\text { ahead. }\end{array}$ & Activity1 & $\begin{array}{l}\text { oscillating } \\
\text { ahead. }\end{array}$ \\
\hline $\begin{array}{c}\text { Arm- } \\
\text { Activity2 }\end{array}$ & $\begin{array}{l}\text { The arm was } \\
\text { oscillating from } \\
\text { straight down to } \\
\text { horizontal (open arm } \\
\text { ). }\end{array}$ & $\begin{array}{c}\text { Leg- } \\
\text { Activity2 }\end{array}$ & $\begin{array}{l}\text { The leg was } \\
\text { oscillating } \\
\text { behind. }\end{array}$ \\
\hline $\begin{array}{c}\text { Arm- } \\
\text { Activity3 }\end{array}$ & $\begin{array}{l}\text { The forearm moved } \\
\text { from straight down to } \\
\text { horizontal straight } \\
\text { ahead. }\end{array}$ & $\begin{array}{c}\text { Leg- } \\
\text { Activity3 }\end{array}$ & $\begin{array}{l}\text { The leg was } \\
\text { performing a } \\
\text { complete } \\
\text { oscillation } \\
\text { ahead- } \\
\text { behind. }\end{array}$ \\
\hline $\begin{array}{c}\text { Leg- } \\
\text { Activity4 }\end{array}$ & $\begin{array}{l}\text { The leg was } \\
\text { oscillating on side. }\end{array}$ & $\begin{array}{c}\text { Leg- } \\
\text { Activity5 } \\
\text { Q }\end{array}$ & $\begin{array}{l}\text { Lifting the } \\
\text { knee. }\end{array}$ \\
\hline
\end{tabular}


CIEEE 2011 This paper is published in the IEEE Transactions on Information Technology in Biomedicine it is available from IEEEXplore at $<\mathrm{http} / / / \mathrm{dx}$.doi.org/10.1109/TITB.2011.2118763>

training dataset where the class of each object is known. After training, the classifier should be able to assign a new object to its right class: in the testing phase the classifier is applied to a testing dataset. By comparing the classifications made on the testing dataset, the performance of the classifier is evaluated.

In our case, each activity (a class) produced two traces (one for each receiver, S1 and S2), each composed of 240 RSS samples. Each pair of traces is an object to be classified.

Most classifiers work in a feature space, which is a multidimensional space where each object is represented by a point. In the feature space, the coordinates of the point are the values of the object's features. A feature can be any quantity that is significant for the object. Usually, features are normalized, so that all points lie in the unity hypercube of the feature space. The most important step in classification problems is the choice of relevant features. The number of features should be as low as possible to avoid overfitting and reduce computational complexity. Their number should be sufficient to distinguish the objects, namely to assign each activity to the right class.

In our case, each object (a pair of RSS traces) was identified by up to five features, as described in the next section. The specific features extracted from the RSS traces were chosen using Weka, a collection of tools for data preprocessing, classification, clustering and more [15]. Weka was also used to compare the performance of two different classifiers working in the same feature space.

K-Nearest Neighbour (K-NN) is a supervised learning algorithm where new objects are classified based on a voting criteria: the $\mathrm{K}$ nearest objects from the training set are considered, and the new object is assigned the class of the majority of those. The training phase of the K-NN algorithm consists in storing the features and the class label of the training objects. In the classification phase, an unlabelled object is classified by assigning the most frequent label among those of the $k$ training samples nearest to it. Various distance metrics can be used, the Euclidean distance being the most common. In this work we used the most basic settings for the algorithm: Euclidean distance and $k$ set to 1 . This means that the class label chosen was the same as the one of the closest training object.

Support Vector Machine (SVM) is a sophisticated learning technique that can deliver good detection and classification performance. In its basic form SVM is a binary linear classifier, meaning that it assumes linear separability of two classes of data and attempts to find a hyperplane in the feature space separating the data points of the two classes. The optimum separation is achieved by the hyperplane that maximizes its distance from the marginal data points on each side (the support vectors), that is the maximum-margin hyperplane. Computation of the hyperplane can be made using quadratic programming, a computationally efficient optimization technique.

The first improvement on the basic form of the SVM is to account for data sets that can not be clearly separated by a hyperplane, by using soft margins. This means that the algorithm chooses a trade-off between a large margin and the possibility of some points being misclassified. The second improvement, which makes the method so powerful, is to map the non linearly separable classes into a high dimensional feature space where the classes become linearly separable using a non-linear kernel function $[16,17]$. What makes this technique computationally efficient is that, by choosing an appropriate kernel function, quadratic programming can still be applied [16,17].

In this work, the classical Pearson kernel function was chosen as one of the best performers. Binary classifiers can be combined to solve multiclass problems. An one-against-one approach was used to tackle the multiclass classification problem. The classification is made by a max-wins voting strategy. A specific classifier is trained for every pair of classes (in our case a class was associated to a specific activity ). For a test sequence, each classifier assigns one vote, and the object is assigned to the class with the highest number of votes.

For both classification methods, classification performance was computed by using a 10-fold cross-validation technique. An object set (a pair of traces, for each exercise) was randomly subdivided into 10 equal-sized partitions: 9 of them were used as the training dataset and the last one was used as the testing dataset. The same procedure was repeated 10 times, until each partition was used for testing. In this way, each object was used exactly once for testing.

\section{Results and Discussions}

\section{A. Preliminary RSS traces analysis}

Fig. 2 presents an example of typical RSS 30 secondsregistrations for arm and leg activities. The movement periodicity is clearly apparent, as well as the variations between RSS traces relevant to different activities.

The activity velocity can be identified using standard methods for fundamental frequency estimation. In Fig. 3, an example of the Fourier transform of an RSS trace is presented, when Activity 1 was performed for 30 seconds at two different rates: 24 oscillations per minute and 72 oscillations per minute. Two spectral components appear at around $0.4 \mathrm{~Hz}$ and $1.2 \mathrm{~Hz}$, for both sensors (S1 and S2), which clearly correspond to the slow rate and fast rate, respectively.

\section{B. Feature extraction}

The first step of the classification procedure was to identify a limited number of features that act as the "fingerprint" of a trace. An initial large set of possible features was defined, from which the best performers were chosen using the feature selection tools provided by Weka. In the set of possible features we considered both time-independent and time-series based statistics. 
CIEEE 2011 This paper is published in the IEEE Transactions on Information Technology in Biomedicine it is available from IEEEXplore at $<\mathrm{http} / / / \mathrm{dx}$.doi.org/10.1109/TITB.2011.2118763>
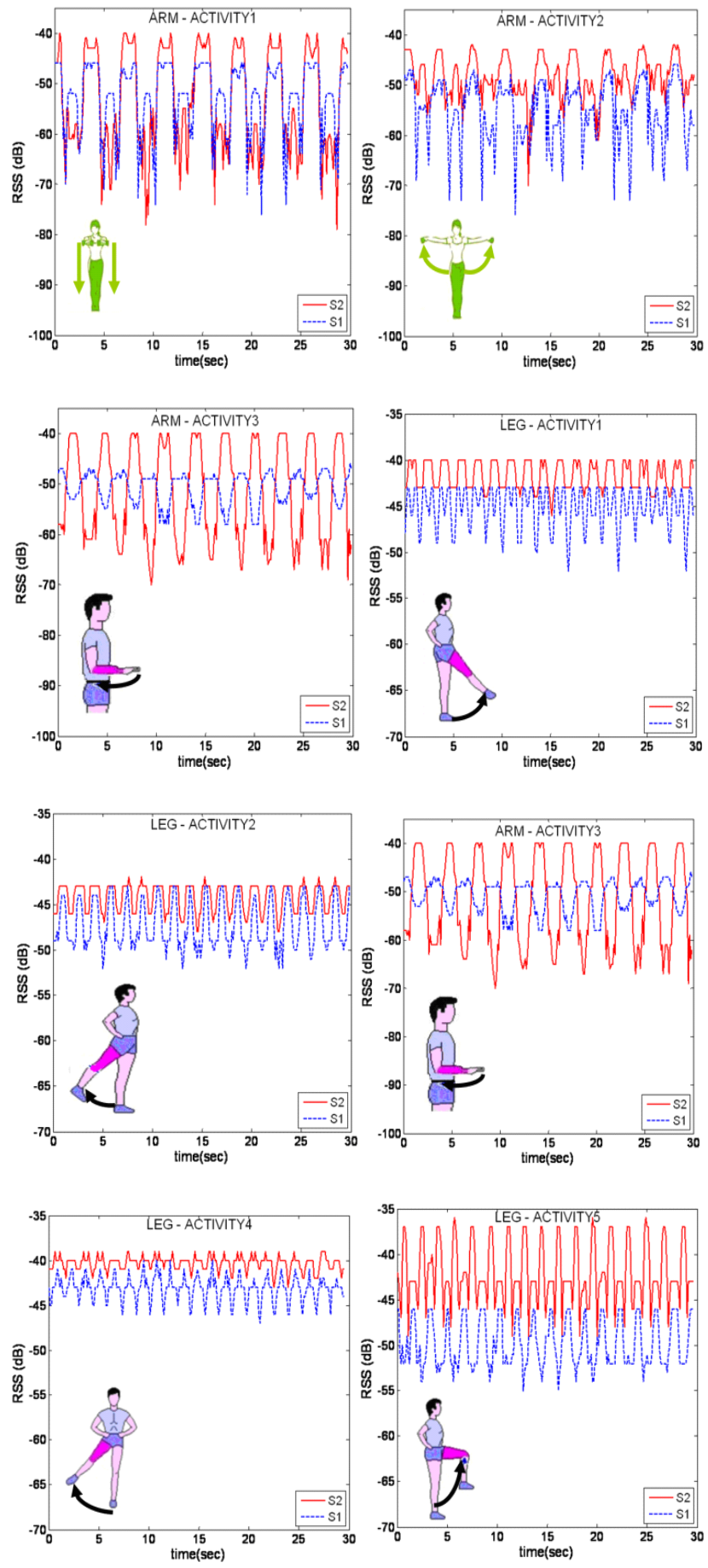

Fig. 2. Samples of RSS traces for arm and leg activities, for both sensors S1 and S2

As far as time-independent statistics are concerned, the ones involving only one transceiver (either S1 or S2) were: mean value $\mu$, standard deviation $\sigma$, skewness, kurtosis. The one involving both transceivers (S1 and S2) was the crosscorrelation $\rho$.

As far as time-series based statistics are concerned, we considered the level crossing rate (LCR) at four different thresholds, firstly computed on both devices separately, and secondly on the difference of the devices' RSS measurements.
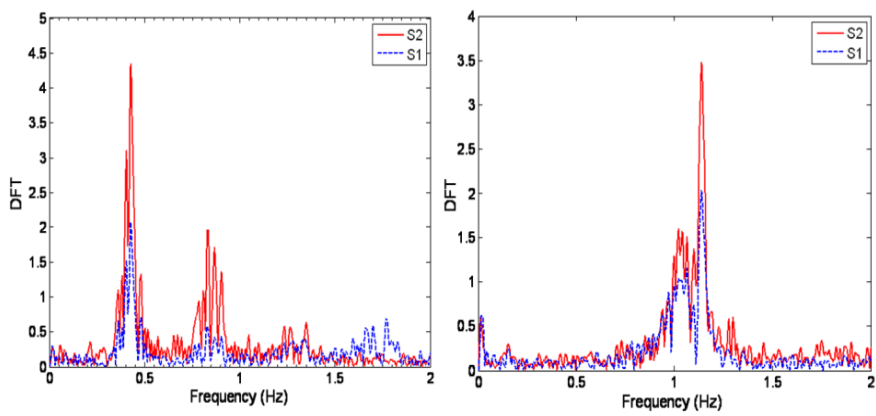

Fig. 3. RSS trace Fourier transform of the arm Activity 1: (a) slow activity (24 oscillations per minute), (b) fast activity (72 oscillations per minute).

The LCR is a statistical parameter that quantifies how often the signal crosses a given threshold in the positivegoing direction. The four thresholds considered in this work were $L C R 1$ at $\mu-0.5 \sigma, L C R 2$ at $\mu+0.5 \sigma, L C R 3$ at $\mu-\sigma$ and $L C R 4$ at $\mu+\sigma$.

A features short list was selected from the initial large set data in order to optimise classification performance. If both transceivers S1 and S2 are used, the list of features includes the mean values, $\mu_{1}$ and $\mu_{2}$, the standard deviations, $\sigma_{1}$ and $\sigma_{2}$, and the correlation coefficient $\rho$. If only one sensor is used, the feature list includes the mean value $\mu$, the standard deviation $\sigma, L C R 1$ and $L C R 2$.

An example of how some features $(L C R 1, \mu, \sigma)$ are distributed is shown in Fig. 4. As shown in Fig. 4a, for the arm exercises, Activity 3 is visually well separated from Activity 1 and Activity 2, which means that Activity 3 can be well recognized. For the leg exercises, Fig. $4 \mathrm{~b}$ shows that Activity 4 and Activity 5 can be easily recognized, whereas activities 1,2 and 3 are more difficult to identify and may be confused with others.

\section{Classification results}

Performance of the proposed system is measured in terms of error rate or, equivalently, of matching rate. The former was defined as the number of misclassifications divided by the total number of trials, while the latter is its complementary (i.e. 1 minus the error rate).

Fig. 5a shows the error rate for the arm as a function of the number of features, for both persons (users A and B) and both SVM and K-NN algorithms. Firstly, two features of the two devices were considered $\left(\mu_{1}\right.$ and $\left.\mu_{2}\right)$ achieving about $70 \%$ and $85 \%$ of matching rate for the users A and B, respectively. The matching rate increases with the number of features, as expected. In fact, when using five features instead of four $\left(\mu_{1}\right.$, $\mu_{2}, \sigma_{1}, \sigma_{2}$ and the fifth feature $\rho$ ) $90 \%$ and $100 \%$ matching rates were achieved for the users $\mathrm{A}$ and $\mathrm{B}$, respectively. In this case correlation $\rho$ does not significantly improve the performance. Performance in terms of error rate shown in Fig. 5a is similar for both SVM and K-NN algorithms.

In order to evaluate which transceiver performs better, the performance of the K-NN algorithm is shown (SVM performance being similar). 
CIEEE 2011 This paper is published in the IEEE Transactions on Information Technology in Biomedicine it is available from IEEEXplore at $<\mathrm{http} / / / \mathrm{dx}$.doi.org/10.1109/TITB.2011.2118763>

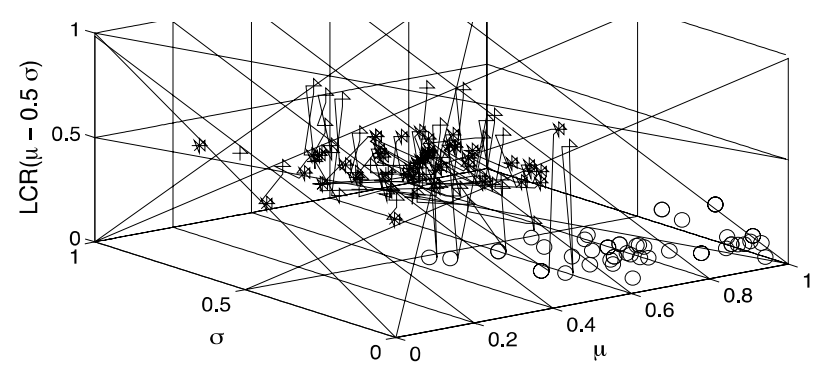

a)

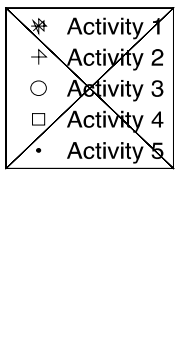

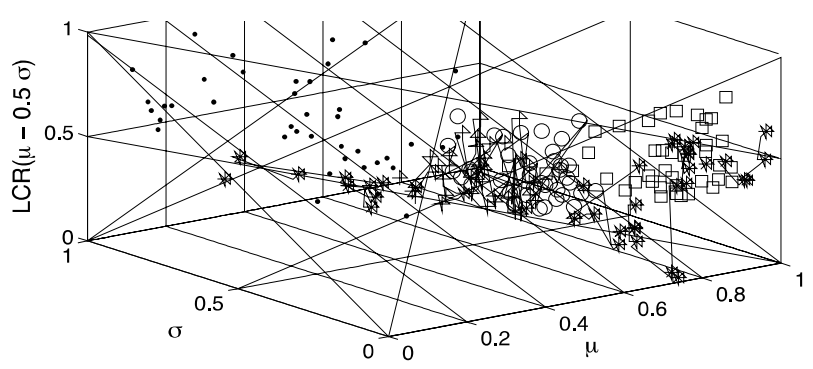

b)
Fig. 5b shows the classification error rate for both users, A and $\mathrm{B}$, for each sensor $\mathrm{S} 1$ and $\mathrm{S} 2$, as a function of the number of features. The features considered were $\mu, \sigma, L C R 1$ and $L C R 2$ for each sensor. Sensor S2 exhibits better performance, probably because it experienced greater oscillations with respect to sensor $\mathrm{S} 1$, and hence greater RSS variations.

Fig. $5 \mathrm{c}$ shows the true positive (TP) and the false positive (FP) rates, considering user $\mathrm{A}$, both algorithms, and five features $\left(\mu_{1}, \mu_{2}, \sigma_{l}, \sigma_{2}, \rho\right)$. For a given activity $\mathrm{A}_{c}$, true positives are the correct classifications with respect to the total number of objects (fifty exercises in our case), while false positives are the erroneous activity classification with respect to the number of times the algorithm chooses $\mathrm{A}_{\mathrm{c}}$. For the arm, Activity 1 exhibited 94\% TP and 20\% FP, Activity 2 was classified with $90 \% \mathrm{TP}$ and $2 \% \mathrm{FP}$, and Activity 3 was classified with $90 \% \mathrm{TP}$ and $4 \% \mathrm{FP}$, when the K-NN algorithm was chosen. Activity 1 presented the highest value of FP, which means that it was the most often misclassified one, but at the same time it had the highest value of TP, making it the most correctly recognized movement. When the SVM algorithm was used, Activity 3 was the most correctly recognized movement, while Activity 1 was the most often misclassified one.

Fig. 5d shows TP and FP rates for user A using four features of sensor S2 $(\mu, \sigma, L C R 1$ and $L C R 2)$ and using both classification algorithms. Activity 3 was always recognized when using K-NN (100\% TP) and was rarely confused with other activities (2\% FP). Activity 1 shows $98 \%$ TP and 7\% FP, while Activity 2 was classified with 97\% TP and never confused with the other activities ( $0 \% \mathrm{FP})$.

The same analysis as in the arm case was performed for the leg case. Specifically, Fig. 5e shows the error rate for the leg as a function of the number of features, for both users and for both algorithms. Firstly, the two features $\mu_{1}$ and $\mu_{2}$ were considered, which achieve about $35 \%$ and $20 \%$ of error rate for the $\mathrm{A}$ and $\mathrm{B}$ users, respectively. When the number of features is increased to five $\left(\mu_{1}, \mu_{2}, \sigma_{l}, \sigma_{2}, \rho\right)$, performance improves. The error rate decreases to about $20 \%$ and $10 \%$ for the A and B users, respectively. Fig. 5f shows the error rates for both users as a function of number of features, for each sensor. The features used for each sensor were $\mu, \sigma, L C R 1$ and $L C R 2$. Due to the similar results achieved with SVM and K$\mathrm{NN}$, only the results obtained with K-NN are shown. As opposed to the arm case, both sensors give similar results.
Fig. 5g shows the TP and FP matching rates for user A when the five features $\mu_{1}, \mu_{2}, \sigma_{l}, \sigma_{2}, \rho$ and both algorithms were used. Activity 5 was always correctly recognized $(100 \%$ $\mathrm{TP})$, and never confused with other ones, ( $0 \% \mathrm{FP})$. This happens probably because it was the only movement significantly different from the other ones.

Fig. 5h shows TP and FP rates for user A using four features $(\mu, \sigma, L C R 1, L C R 2)$ of sensor S2. Results for both classification algorithms are shown. Also in this case, Activity 5 was always recognized (100\% TP) and never confused with other activities ( $0 \% \mathrm{FP})$.

Finally, confusion matrices for the analyzed classification problem are presented in Fig. 6. Confusion matrices are a compact graphical representation where each row of the matrix corresponds to the activity assigned by the classifier (predicted class), while each column represents the activity performed (actual class).

A classification method with ideal performance will only have bars on the main diagonal of the matrix. The more bars on the non-diagonal cells are high, the worst the classification performance. As far as the arm exercises are concerned, for SVM and the two-features case $\left(\mu_{1}\right.$ and $\left.\mu_{2}\right)$ there was moderate confusion between Activity 1 and Activity 2. In fact, $46 \%$ Activity 2 was misclassified as Activity 1, and about $10 \%$ were misclassified as Activity 3 .

On the other hand, Activity 1 and Activity 3 were quite well recognized even with only two features. Activity 2 was less recognized probably because the antenna position of the transceiver S2 is more in a Line of Sight region (LOS) with respect to the other cases. As expected, the greater the number of features, the less the error rate. No more than $8 \%$ of the Activity 1 was misclassified as Activity 2 and vice versa. K-NN and SVM algorithms behave similarly.

As far as the leg exercises are concerned, for both SVM and K-NN, Activity 4 and Activity 5 were very well recognized and not confused with other leg movements, even for only two selected features. On the other hand, the first three activities were misclassified, probably because they are quite similar to each other. Increasing the number of features the rate of misclassification decrease, as expected. Specifically, for K-NN and five features, about $17 \%$ of Activity 3 was misclassified as Activity 2, and $7 \%$ as Activity 1. 
CIEEE 2011 This paper is published in the IEEE Transactions on Information Technology in Biomedicine it is available from IEEEXplore at $<$ http://dx.doi.org/10.1109/TITB.2011.2118763>

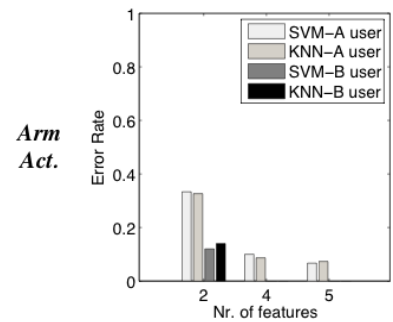

a)

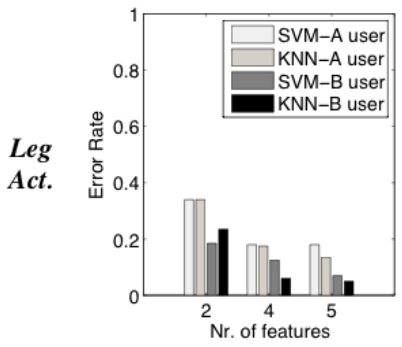

e)

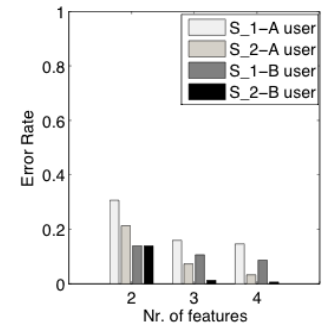

b)

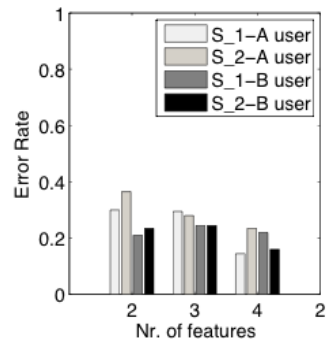

f)

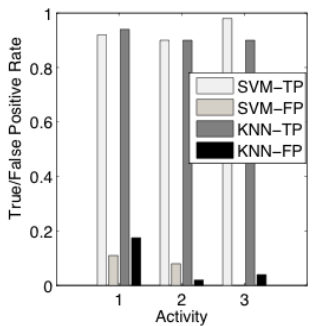

c)

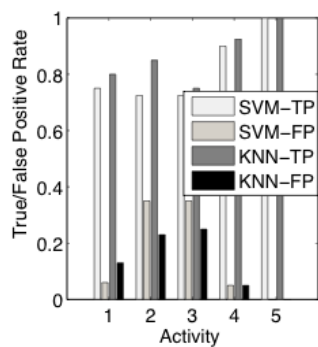

g)
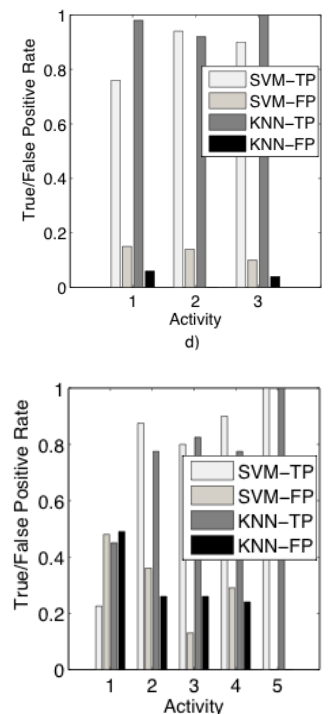

h)

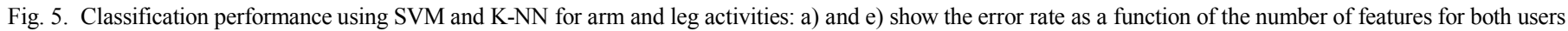

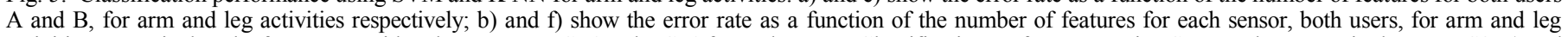

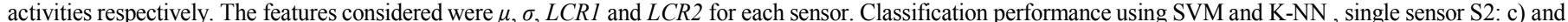

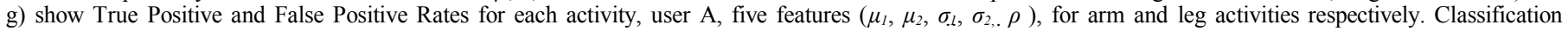

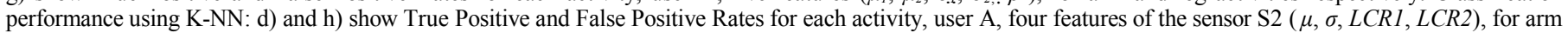
and leg activities respectively.

Similarly to the arm case, when Activity 2 was performed, the antenna position of the transceiver S1 was closer to a nonLOS condition, which may be the cause for a misclassification of Activity 3 as Activity 2 and Activity 1.

The consequences of transceivers not being in sight of each other needs further investigation: while passing from a LOS to a non-LOS condition during exercises might be beneficial, as it has the potential of amplifying the RSS variations during the movement, complete lack of LOS during the whole exercise may be detrimental, because it has potential for lowering the overall strength of received signal and its information content. These considerations, however, are not final and further studies are necessary on the optimal placement of transceivers on body and the outcomes of suboptimal placement.

\section{Conclusions}

Automatic recognition of some arm and leg exercises was addressed for use in orthopedic physical therapy: measurements showed that it is possible to use low-cost transceivers to classify limb activities. Good classification performance can be achieved by using only the received signal strength measurements relevant to three wearable wireless sensors. A simple K-NN classifier and a more sophisticated SVM classifier yielded similar performance, suggesting that the choice of the classifier is not critical. The features considered for classification are computationally inexpensive and only a few (three to five) were sufficient to obtain a good identification accuracy, even for relatively similar movements.

The main benefit of using received signal strength measurements is that they are readily available on small wireless communication devices. We envision that these are going to be extensively deployed in indoor environments in the near future, for communication and control purposes. The idea is to exploit the ubiquity of wireless sensors to obtain measurements "for free". The outcome of this feasibility study is twofold. Firstly, it is possible to recognize human limb movements without the need of ad hoc sensors, provided that wireless transceivers are already installed on the human body. Secondly, if dedicated sensors are already installed for this purpose, their results can be complemented with RSS measurements, thus potentially improving accuracy and reliability.

\section{ACKNOWLEDGMENTS}

The authors wish to thank Stefano Lenzi, Sara Marongiu and Francesco Crudo for their help in the measurement campaign.

\section{REFERENCES}

[1] V. Shnayder, B. Chen, K. Lorincz, T. R. F. Fulford-Jones, M. Welsh, "Sensor Networks for Medical Care", Technical Report, Harvard University, 2005

[2] Q. Wang, W. Shin, X. liu, Z. Zeng, C. Oh, B. K. AlShebli, M. Caccamo, C.A. Gunter, E. Gunter, J. Hou, K. Karahalios, L. Sha, "I-Living: An open system architecture for assisted living", IEEE International Conference on Systems, Man and Cybernetics, October 2006.

[3] M. Annavaram, N. Medvidovic, U. Mitra, S. Narayanan, G. Sukhatme, Z. Meng, S. Qiu, R. Kumar, G. Thatte, D. Spruijt-Metz, "Multimodal sensing for pediatric obesity applications" International Workshop on Urban, Community, and Social Applications of Networked Sensing Systems, November 2008.

[4] Tanmay Pawar, Subhasis Chaudhuri, Siddhartha P. Duttagupta, "Body movement activity recognition for ambulatory cardiac monitoring", IEEE Transactions on Biomedical Engineering, vol. 54, no. 5, p.874-882, May 2007.

[5] P. Iso-Ketola, T. Karinsalo, L. Vanhala, "HipGuard: A wearable measurement system for patients recovering from a hip operation", Proceedings of the Pervasive Computing Technologies for Healthcare Conference, February 2008. 
CIEEE 2011 This paper is published in the IEEE Transactions on Information Technology in Biomedicine it is available from IEEEXPlore at $<$ http://dx.doi.org/10.1109/TITB.2011.2118763>

2 features

Arm
Act.
SVM

Arm

Act.

KNN

Leg

Act.

$S V M$

Leg

Act.

KNN
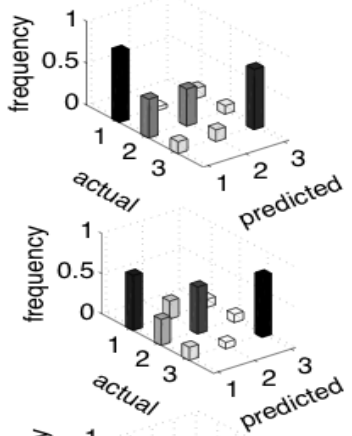

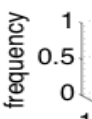
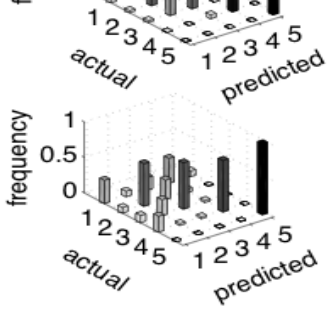

0.10 .8
4 features
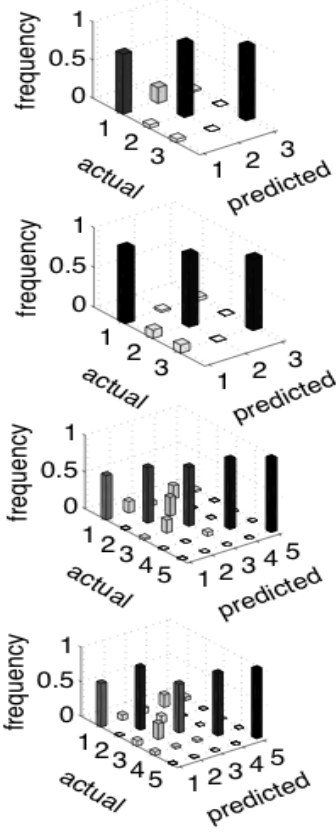

5 features
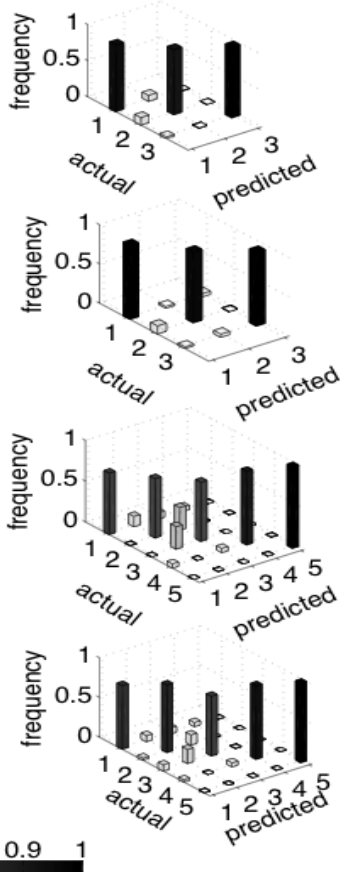

Fig. 6. Confusion matrices for the limb movements. The two axes on the base of each graph represent the actual activity class and the class predicted by the algorithm, respectively. The performance is given in a matrix fashion; four rows (arm and leg activities for each classification method) and three columns (for each selected features). The smaller the bars outside of the main diagonal, the better the performance.

[6] K.D. Nguyen, I.M. Chen, Z. Luo, S.H. Yeo, H.L. Duh, “A wearable sensing system for tracking and monitoring of functional arm movement", IEEE/ASME Transactions on Mechatronics, vol. PP, no. 99, p. 1-8, February 2010.

[7] K. Lorincz, B. Chen, G. W. Challen, A. R. Chowdhury, S. Patel, P. Bonato, M. Welsh, "Mercury: A wearable sensor network platform for high-fidelity motion analysis", Embedded Networked Sensor Systems Conf.(SenSys'09), 4-6 November 2009.

[8] J. Baek, G. Lee, W. Park, B-J. Yun, "Accelerometer Signal Processing for user activity detection", Book Chapter, Knowledge-Based Intelligent Information and Engineering Systems, p. 610-617, 2004.

[9] M. Galli, C. Rigoldi, L. Mainardi, N. Tenore, P. Onorati, G. Albertini, "Postural control in patients with Down syndrome", Disability \& Rehabilitation, vol. 30, no. 30 (17), p. 1274-1278, 2008.

[10] A. M. Adami, M. Pavel, T. L. Hayers, C. M. Singer, "Detection of movement in bed using unobtrusive load cell sensors", IEEE Trans. On Information Technology in Biomedicine, vol. 14, no. 2, p.481-490, March 2010.

[11] http://www.xbow.com

[12] H. Y. Lau, K. Y. Tong, H. Zhu, "Support vector machine for classification of walking condition using miniature kinematic sensors", Med. Biol. Eng. Comput., vol. 46, no. 6, p. 563-573, June 2008.

[13] S. Patel, K. Lorincz, R. Hughes, N. Huggins, J. Growdon, D. Standaert, M. Akay, J. Dy, M. welsh, P. Bonato, "Monitoring motor fluctuations in patients with Parkinson's disease using wearable sensors", IEEE Trans. On Information Technology in Biomedicine, vol. 13, no. 6, November 2009.

[14] S. Biswas, M. Quwaider, "Body posture identification using Hidden Markov Model with wearable sensor networks", International Conference on Body Area Networks, March 2008.

[15] Mark Hall, Eibe Frank, Geoffrey Holmes, Bernhard Pfahringer, Peter Reutemann, Ian H. Witten, "The WEKA Data Mining Software: An Update; SIGKDD Explorations, vol.11, no.1, p. 10-18, 2009.

[16] C. Cortes, V. Vapnik, "Support-Vector Networks", Machine Learning, vol.20, p. 273-297, 1995.

[17] S.R. Glenn, "Support Vector Machines for Classification and Regression", Technical Report, May 1998.
Anda R. Guraliuc received the BS and MS degree from the University of Bucharest, Romania, in 2003 and 2005, respectively. She also received the $\mathrm{PhD}$ degree from the University of Pisa in 2008. Since 2008 she is Research Associate at the Microwave and Radiation Laboratory, Department of Information Engineering, University of Pisa, Italy. Her research interests include antenna design and on-body communication systems.

Paolo Barsocchi received his MSc and $\mathrm{PhD}$ degrees in Information Engineering from the University of Pisa, Italy, in 2003 and 2007, respectively. Since 2004 he is Assistant Researcher at the ISTI/CNR Institute. His research interests are related to wireless mobile systems and architectures, wireless channel modeling, and indoor localization in AAL environment.

Francesco Potortì received his engineering degree from the University of Pisa (IT) in 1991. Currently, he is a Senior Researcher at the ISTI institute of CNR. His research interests include wireless communications, internet technology, simulation of communications systems and RSSI-based indoor localisation. He is co-authored of more than fifty refereed scientific papers.

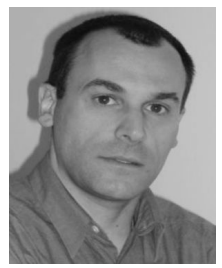

Paolo Nepa (M'08) received the Laurea (Doctor) degree in electronics engineering (summa cum laude) from the University of Pisa, Pisa, Italy, in 1990. Currently, he is Associate Professor at the University of Pisa, Italy. His research interests include the extension of high-frequency techniques to electromagnetic scattering from material structures and the design of wideband and multiband antennas for base stations and mobile terminals of wireless communication systems. He is also involved in the analysis and design of diversity schemes for mobile terminals and on-body communication systems. He received the Young Scientist Award from the International Union of Radio Science in 1998. 